\title{
Dynamic Response of Parallel Hoisting System under Drive Deviation between Ropes with Time-Varying Length
}

\author{
Guohua Cao, ${ }^{1,2}$ Xiang Cai, ${ }^{1,2}$ Naige Wang, ${ }^{1,2}$ Weihong Peng, ${ }^{3}$ and Jishun Li $^{4}$ \\ ${ }^{1}$ School of Mechatronic Engineering, China University of Mining and Technology, Xuzhou 221116, China \\ ${ }^{2}$ Jiangsu Key Laboratory of Mine Mechanical and Electrical Equipment, China University of Mining and Technology, \\ Xuzhou 221116, China \\ ${ }^{3}$ School of Mechanics and Civil Engineering, China University of Mining and Technology, \\ Xuzhou 221116, China \\ ${ }^{4}$ State Key Laboratory of Heavy Mining Equipment, Luoyang 471039, China \\ Correspondence should be addressed to Guohua Cao; caoguohua@cumt.edu.cn
}

Received 12 September 2016; Revised 6 December 2016; Accepted 21 December 2016; Published 29 January 2017

Academic Editor: Carlo Trigona

Copyright @ 2017 Guohua Cao et al. This is an open access article distributed under the Creative Commons Attribution License, which permits unrestricted use, distribution, and reproduction in any medium, provided the original work is properly cited.

\begin{abstract}
The dynamic responses of parallel hoisting system with time-varying length and rigid guidance under drive deviation are investigated considering tension and torsion characteristics of the ropes. The variable-domain three-node elements of rope are employed and the corresponding differential algebraic equations (DAEs) are derived using Lagrange's equations of the first kind. The slack situation of the rope is considered, and the dynamic equations which are systems of DAEs are transformed to ordinary differential equations (ODEs). The dynamic responses of tension, torsion, and acceleration are analyzed considering radius' error of the drums, which indicates that the drive deviation between ropes can cause large influence on the tension difference and even cause one of the ropes to slack. However, the torsion of the corresponding rope is active. And unreasonable discordance between ropes should be controlled for the design and manufacture of drum on super deep parallel hoisting system.
\end{abstract}

\section{Introduction}

Parallel hoisting system, which has the advantage of large carrying capacity, small rotation of conveyance, excellent security performance, and fine economic performance, works at the manner that one conveyance is hoisted cooperatively by several cables [1]. Parallel hoisting system is widely used due to the increase of lifting heavy and large volume loads, such as elevator hoisting system (Figure 1(a)), mine hoisting system (Figure 1(b)), and parallel suspension platform of vertical shaft in construction.

The parallel hoisting system will vibrate subject to the excitation sources including ripple of the motor, turbulence of transmission device, abrasion of winding drum, and the eccentricity of head sheave. These vibrations will affect the stability of the conveyance and may also cause damage to the ropes, which will further lead to accidents.

Dynamic characteristics of single rope hoisting system with time-varying length have been studied by many researchers. Zhu and Ni investigated the linear lateral dynamics of beam and string with an arbitrarily varying length by Hamilton's principle and Galerkin's method [2]. Kaczmarczyk and Ostachowicz described the coupled laterallongitudinal dynamic response of the cables in deep mine hoisting system with a depth of $2085 \mathrm{~m}$ and formulated a discrete mathematical model by Rayleigh-Ritz procedure [3]. Wang et al. investigated the lateral response of the moving hoisting conveyance in cable-guided hoisting system [4]. All of these researches in hoisting system with single cable are the foundations for the dynamic research of parallel hoisting system.

As the application of parallel hoisting systems is increasing, people are paying more and more attention on its research. Zhu and Ren developed a spatial discretization and substructure method to accurately calculate the dynamic responses of single and multiple suspension cables with length-variant distributed-parameter components, which 


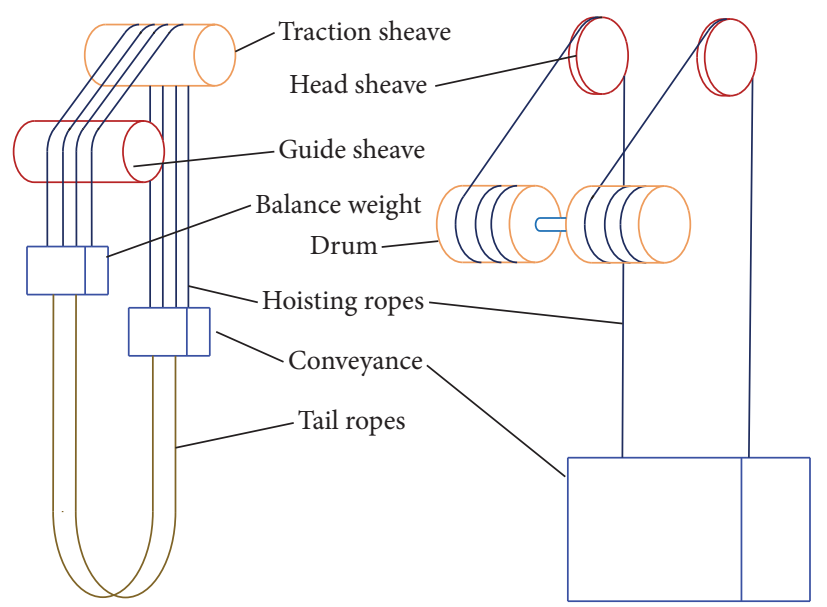

(a) Elevator hoisting system

Figure 1: Parallel hoisting system.

simplified the handle of boundary conditions [5]. References [6-9] investigated the dynamics of cable-suspended parallel system, but the vibrations of cables themselves are not considered. So, it could not get accurate results. Shao et al. investigated the dynamics of a sinking winch mechanism in the framework of nonsmooth dynamics considering the unilateral property of cable and presented a numerical simulation method which is suitable for the dynamic analysis of the sinking winch mechanism [10]. Du et al. addressed the dynamic modeling of large CDPMs using a variable-domain finite element method; the effects of cable length variation and the resulting mass variation are also considered [1]. Wang et al. established the longitudinal vibration model of parallel hoisting system with tension autobalance device attached to the ends of all hoisting ropes and showed the influence on longitudinal response of different coefficients and excitations [11].

Finite element method (FEM), as a well-known and efficient numerical method, has been widely used in engineering problems [12]. It can be used to deal with the dynamic problem of continuum [13]. Stylianou and Tabarrok analyzed the dynamic characteristic of an axially moving beam $[14,15]$. Wang et al. investigated three-dimensional vibrations of an underwater geometrically nonlinear cable with a weight at the lower end [16]. Moustafa et al. considered the modeling problem of the dynamics of overhead cranes with flexible cable and load hoisting or lowering during crane travel [17]. All these researches are investigated by variable-domain FEM (Vd-FEM). The Vd-FEM does not need to find trial functions to satisfy the boundary conditions while shape functions are used as interpolation functions. And the displacement boundary conditions at upper ends of cables can be satisfied accurately by modifying the global matrix properly.

Wire rope, for its complex helical structure, will produce twist motion or torsion under axial load. Costello derived the coupled stiffness coefficient of wire rope, which can be used to express the relation between axial force and torsion with strain [18]. Thus, when ropes vibrate longitudinally, the torsional vibrations will also occurred. The different upper ends

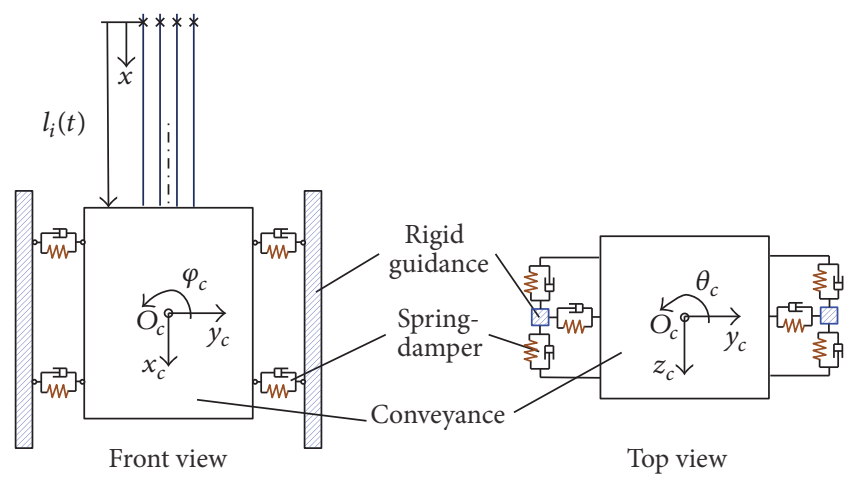

FIgURE 2: Model of parallel hoisting system.

excitations will cause different tension and torsion of ropes. The torsion difference will cause the conveyance rotating around the vertical axis, and tension difference in ropes also causes the conveyance rotating around the horizontal axis. Sometimes the rotation of conveyance can be uncomfortable for the passengers or even damage the guide devices. In this paper, the dynamic responses of parallel hoisting system with time-varying length and rigid guidance under drive deviation are investigated considering tension and torsion characteristics of ropes. The variable-domain three-node elements of rope are used and the corresponding differential algebraic equations (DAEs) are derived by Lagrange's equations of the first kind, which are for working out several challenging problems including the tensions and torsions between the conveyance and cables and the conveyance motion. The dynamic responses of parallel hoisting system are discussed by considering radius' error of drum.

\section{Model for Parallel Hoisting System}

2.1. Description of Parallel Hoisting System. Parallel hoisting system depicted in Figure 1 can be simplified as $k$ parallel ropes with an attached conveyance which is restrained by rigid guidance, where $k$ is the number of hoisting ropes, as shown in Figure 2. Length of each rope is $l(t)$ at time $t$ excluding the excitation at upper end. The vertically translating velocity and acceleration of ropes are $v(t)=\dot{l}(t)$ and $a(t)=\ddot{l}(t)$, where the overdot denotes time differentiation, and the same representation is used as follows.

$e_{i}(t)$ represents the longitudinal drive deviation on $i$ th rope at upper end, which might be caused by the radius' error or irregular outline of drum. $\theta_{c}$ and $\varphi_{c}$ denote the angle displacements of conveyance about vertical axis $x_{c}$ and horizontal axis $z_{c}$ as shown in Figure 2. The following assumptions constrain the analysis. (1) Guidance devices are always keeping in touch with rigid guidance under preload; (2) the whole friction can be neglected; (3) the influence of lateral vibration of rope on the system is small enough to be neglected; (4) the ropes' mechanical parameters remain constant.

2.2. Equations of Motion. The kinetic energy of the $k$ ropes and conveyance is given by 

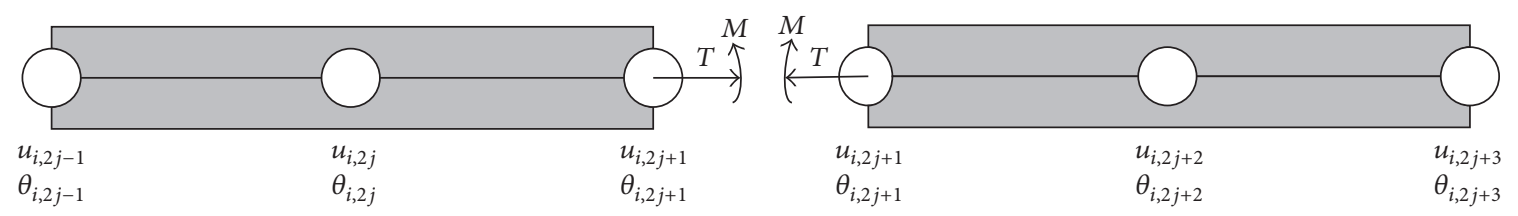

Figure 3: Three-node element of rope.

$$
\begin{aligned}
T= & \sum_{i=1}^{k} \sum_{j=1}^{n} \frac{1}{2} \int_{(j-1) l(t) / n}^{j l(t) / n} \rho\left(\frac{D u_{i}}{D t}+v\right)^{2} \mathrm{~d} x \\
& +\sum_{i=1}^{k} \sum_{j=1}^{n} \frac{1}{2} \int_{(j-1) l(t) / n}^{j l(t) / n} J\left(\frac{D \theta_{i}}{D t}\right)^{2} \mathrm{~d} x \\
& +\frac{1}{2} m_{c}\left(\dot{u}_{c}+v\right)^{2}+\frac{1}{2} J_{c x} \dot{\theta}_{c}^{2}+\frac{1}{2} J_{c z} \dot{\varphi}_{c}^{2},
\end{aligned}
$$

where $n$ is the number of elements in each rope, $\rho$ is density of hoisting rope per unit length, $J$ is the moment of inertia of hoisting rope about its central axis, and $j$ denotes the $j$ th element of the ropes. $m_{c}, J_{c x}$, and $J_{c z}$ are mass and the moment of inertia about its vertical and horizontal axis of conveyance, respectively. $u_{c}$ is the dynamic longitudinal displacement of conveyance. The operator $D / D t$ is given by $D / D t=\partial / \partial t+$ $v(\partial / \partial x)$.

In (1), $u_{i}$ and $\theta_{i}$ are given as

$$
\begin{aligned}
u_{i}(x, t) & =\mathbf{N}_{j}(x, l(t)) \mathbf{q}_{i, j}(t), \\
\theta_{i}(x, t) & =\mathbf{N}_{j}(x, l(t)) \mathbf{p}_{i, j}(t), \\
x_{j} & \leq x \leq x_{j+1}, j=1,2, \ldots, n,
\end{aligned}
$$

where $\mathbf{q}_{i, j}(t)=\left[\begin{array}{lll}u_{i, 2 j-1} & u_{i, 2 j} & u_{i, 2 j+1}\end{array}\right]^{\mathrm{T}}$ and $\mathbf{p}_{i, j}(t)=$ $\left[\begin{array}{lll}\theta_{i, 2 j-1} & \theta_{i, 2 j} & \theta_{i, 2 j+1}\end{array}\right]^{\mathrm{T}}$ are longitudinal and torsional displacement vectors, $\mathbf{N}_{j}(x, l)=\left[\begin{array}{lll}N_{j 1} & N_{j 2} & N_{j 3}\end{array}\right]$ is shape function matrix depending on $l(t)$, where $N_{j 1}=2 j^{2}-j+$ $n x(1-4 j) / l+2(n x / l)^{2}, N_{j 2}=4\left[j-j^{2}+n x(2 j-1) / l-(n x / l)^{2}\right]$, and $N_{j 3}=1-3 j+2 j^{2}+n x(3-4 j) / l+2(n x / l)^{2}$.

The $j$ th and $(j+1)$ th elements of $i$ th rope are shown in Figure 3, in which $T$ and $M$ are the tension and torsion between the two elements.

Considering the dynamic displacement and static elastic deformation, the strain energy of the hoisting system is formulated as

$$
\begin{aligned}
& E_{e} \\
& =\sum_{i=1}^{k} \sum_{j=1}^{n} \int_{(j-1) l(t) / n}^{j l(t) / n}\left[\left(T_{i}^{c}+\frac{1}{2} T_{i}^{d}\right) \varepsilon_{i}+\left(M_{i}^{c}+\frac{1}{2} M_{i}^{d}\right) \phi_{i}\right] \mathrm{d} x \\
& \quad+8 \cdot \frac{1}{2} k_{c}\left(r_{1} \theta_{c}\right)^{2}+4 \cdot \frac{1}{2} k_{c}\left(r_{2} \varphi_{c}\right)^{2},
\end{aligned}
$$

where $\varepsilon_{i}=u_{i, x}$ and $\phi_{i}=\theta_{i, x}$ denote the longitudinal and torsional strain, respectively, where the subscript $x$ denotes partial differentiation with respect to $x$, and the same representation is used as follows. The static tension and torsion in $i$ th rope can be expressed as

$$
\begin{aligned}
T_{i}^{c} & =\rho(l(t)-x) g+\frac{m_{c} g}{k}, \\
M_{i}^{c} & =\frac{T_{i}^{c} Q_{i}^{c}}{Q_{i}^{a}} .
\end{aligned}
$$

The dynamic tension and torsion can be given, respectively, as

$$
\begin{gathered}
T_{i}^{d}=Q_{i}^{a} \varepsilon_{i}+Q_{i}^{b} \phi_{i}, \\
M_{i}^{d}=Q_{i}^{c} \varepsilon_{i}+Q_{i}^{d} \phi_{i}
\end{gathered}
$$

in which $Q_{i}^{a}$ and $Q_{i}^{d}$ denote longitudinal and torsional stiffness coefficients of $i$ th rope, respectively. $Q_{i}^{b}$ and $Q_{i}^{c}$ denote the coupled stiffness coefficient [18]. $k_{c}$ is the equivalent stiffness of guide devices; $r_{1}$ is the horizontal distance between the center of conveyance and rigid guidance; $r_{2}$ is the vertical distance between guide devices and the center of conveyance.

Setting the initial position as the place of zero gravitational potential energy, the gravitational potential energy of system can be written as

$$
E_{g}=-\sum_{i=1}^{k} \sum_{j=1}^{n} \int_{(j-1) l(t) / n}^{j l(t) / n} \rho g u_{i} \mathrm{~d} x-m_{c} g u_{c} .
$$

According to the viscoelastic properties of wire rope [19], the equivalent external nodal load on the $j$ th element of $i$ th rope is formulated as

$$
\begin{aligned}
\mathbf{f}_{e u j} & =\int_{(j-1) l(t) / n}^{j l(t) / n} f_{u c} \mathbf{N}_{j}^{T} \mathrm{~d} x, \\
\mathbf{f}_{e \theta j} & =\int_{(j-1) l(t) / n}^{j l(t) / n} f_{\theta c} \mathbf{N}_{j}^{T} \mathrm{~d} x,
\end{aligned}
$$

where $f_{u c}=\zeta_{u}\left(Q_{i}^{a} \varepsilon_{i, t}+Q_{i}^{b} \phi_{i, t}\right), f_{\theta c}=\zeta_{\theta}\left(Q_{i}^{c} \varepsilon_{i, t}+Q_{i}^{d} \phi_{i, t}\right)$, and $\zeta_{u}$ and $\zeta_{\theta}$ are longitudinal and torsional damping coefficients, respectively.

The global equivalent external nodal load can be obtained as $\sum \mathbf{f}_{e u j}$ and $\sum \mathbf{f}_{e \theta j}$, where $\sum$ denotes the assembly operator of the Vd-FEM.

The damping force of guide device on conveyance is given as

$$
\begin{aligned}
& F_{e \theta c}=-8 c_{c} r_{1} \dot{\theta}_{c}, \\
& F_{e \varphi c}=-4 c_{c} r_{2} \dot{\varphi}_{c},
\end{aligned}
$$

where $c_{c}$ is equivalent damping of guide devices. 
Since $\varphi_{c}$ is very small, it can be obtained approximately by the dynamic displacement of the lower ends of ropes as

$$
g_{1}=\varphi_{c}-\frac{\left(u_{1,2 n+1}-u_{2,2 n+1}\right)}{d}=0,
$$

where $d$ is the distance between adjacent ropes.

The geometric relationships between dynamic longitudinal displacement of conveyance and $k$ ropes at lower ends are given as

$$
\begin{aligned}
& g_{2}=u_{c}-\left(u_{1,2 n+1}-\frac{d \varphi_{c}(k-1)}{2}\right)=0, \\
& g_{i}=u_{i, 2 n+1}-\left(u_{1,2 n+1}-(i-1) d \varphi_{c}\right)=0, \\
& \qquad i=3,4, \ldots, k .
\end{aligned}
$$

The dynamic torsional displacements of lower ends of ropes can be expressed by the rotation angle of conveyance as

$$
g_{k+i}=\theta_{i, 2 n+1}-\theta_{c}=0, \quad i=1,2, \ldots, k .
$$

Substituting (1)-(10) into Lagrange's equations of the first kind [20]

$$
\frac{d}{\mathrm{~d} t} \frac{\partial T}{\partial \dot{Q}_{i}}-\frac{\partial T}{\partial Q_{i}}+\frac{\partial\left(E_{e}+E_{g}\right)}{\partial Q_{i}}=F_{e i}+\sum_{j=1}^{2 k} \lambda_{j} \frac{\partial g_{j}}{\partial Q_{i}}
$$

and using a standard assembly procedure of the FEM [12], the dynamic equations of $k$ ropes and conveyance without the excitations at upper ends can be obtained as

$$
\begin{aligned}
\mathbf{M Q}+\mathbf{C} \dot{\mathbf{Q}}+\mathbf{K Q} & =\mathbf{F}+\mathbf{G}^{T} \boldsymbol{\lambda}, \\
\mathbf{g}(\mathbf{Q}, t) & =\mathbf{0}
\end{aligned}
$$

which is a system of DAEs, where $\mathbf{Q}=\left[u_{1,1}, \theta_{1,1}, u_{2,1}, \theta_{2,1}, \ldots\right.$, $\left.u_{k, 2 n+1}, \theta_{k, 2 n+1}, u_{c}, \theta_{c}, \varphi_{c}\right]^{\mathrm{T}}$ is the displacement vector of all the nodes on $k$ ropes and three degrees of freedom of conveyance.

$\mathbf{M}=\left[\begin{array}{ll}\mathbf{M}_{D} & \\ & \mathbf{M}_{c}\end{array}\right], \mathbf{C}=\left[\begin{array}{ll}\mathbf{C}_{D} & \\ & \mathbf{C}_{c}\end{array}\right], \mathbf{K}=\left[\begin{array}{ll}\mathbf{K}_{D} & \\ & \mathbf{K}_{c}\end{array}\right]$, and $\mathbf{F}=\left[\begin{array}{ll}\mathbf{F}_{D}^{\mathrm{T}} & \mathbf{F}_{c}^{\mathrm{T}}\end{array}\right]^{\mathrm{T}}$ are the matrixes of mass, damping, stiffness, and force. $\mathbf{M}_{D}, \mathbf{C}_{D}, \mathbf{K}_{D}$, and $\mathbf{F}_{D}$ are the global matrixes of ropes assembled from element matrixes, which are listed in the Appendix. And $\mathbf{M}_{c}=\operatorname{diag}\left(m_{c}, J_{c x}, J_{c z}\right), \mathbf{C}_{c}=$ $\operatorname{diag}\left(0,8 c_{c} r_{1}^{2}, 4 c_{c} r_{2}^{2}\right), \mathbf{K}_{c}=\operatorname{diag}\left(0,8 k_{c} r_{1}^{2}, 4 k_{c} r_{2}^{2}\right)$, and $\mathbf{F}_{c}=$ $\left[\begin{array}{lll}m_{c}(g-a) & 0 & 0\end{array}\right]^{\mathrm{T}} \cdot \mathbf{g}(\mathbf{Q}, t)$ is a $2 k$-order vector which includes the constraint equations (11)-(13); $\mathbf{G}=\partial \mathbf{g} / \partial \mathbf{Q}, \boldsymbol{\lambda}=$ $\left[\begin{array}{llll}\lambda_{1} & \lambda_{2} & \cdots & \lambda_{2 k}\end{array}\right]^{\mathrm{T}}$ are Lagrange multipliers.

Considering the following DAEs calculation, according to (11)-(13), the $2 k \times[2 k(2 n+1)+3]$ matrix $\mathbf{G}$ can be written as $\mathbf{G}=\left[\begin{array}{ll}\mathbf{G}_{0} & \mathbf{G}_{1}\end{array}\right]$, where $\mathbf{G}_{0}$ is $2 k \times(4 n k+3)$ matrix and $\mathbf{G}_{1}$ is $2 k \times 2 k$ matrix.

Rewriting the displacement vector as $\mathbf{Q}=\left[\begin{array}{ll}\mathbf{Q}_{0}^{\mathrm{T}} & \mathbf{Q}_{1}^{\mathrm{T}}\end{array}\right]^{\mathrm{T}}$, where $\mathbf{Q}_{0}$ is a $4 n k+3$ vector and $\mathbf{Q}_{1}$ is a $2 k$ vector, hence, $\mathbf{Q}$ can be expressed by $\mathbf{Q}_{0}$ as

$$
\mathbf{Q}=\mathbf{U Q}_{0}
$$

where $\mathbf{U}=\left[\begin{array}{c}\mathbf{I} \\ -\mathbf{G}_{1}^{-1} \mathbf{G}_{0}\end{array}\right]$ and $\mathbf{I}$ is $4 n k+3$ identity matrix.
Differentiating (17) twice with respect to $t$ yields

$$
\begin{aligned}
& \dot{\mathbf{Q}}=\mathbf{U}_{\mathbf{Q}}, \\
& \ddot{\mathbf{Q}}=\mathbf{U} \ddot{\mathbf{Q}}_{0} .
\end{aligned}
$$

Substituting (17) and (18) into (15) and premultiplying it by $\mathbf{U}^{\mathrm{T}}$, the dynamic equations of ropes and conveyance without excitations at upper ends are formulated as

$$
\widetilde{\mathbf{M}} \ddot{\mathbf{Q}}_{0}+\widetilde{\mathbf{C}} \dot{\mathbf{Q}}_{0}+\widetilde{\mathbf{K}} \mathbf{Q}_{0}=\widetilde{\mathbf{F}}
$$

which is a system of ordinary differential equations (ODEs), where $\widetilde{\mathbf{M}}=\mathbf{U}^{\mathrm{T}} \mathbf{M U}, \widetilde{\mathbf{C}}=\mathbf{U}^{\mathrm{T}} \mathbf{C} \mathbf{U}, \widetilde{\mathbf{K}}=\mathbf{U}^{\mathrm{T}} \mathbf{K U}$, and $\widetilde{\mathbf{F}}=\mathbf{U}^{\mathrm{T}} \mathbf{F}$.

When longitudinal excitations $u_{i, 1}=e_{i}(t)$ and torsional constraint $\theta_{i, 1}=0$ are imposed on the upper ends of ropes, the dynamic equations should be converted into the form as follows:

$$
\overline{\mathbf{M}} \ddot{\mathbf{Q}}_{0}+\overline{\mathbf{C}} \dot{\overline{\mathbf{Q}}}_{0}+\overline{\mathbf{K}} \overline{\mathbf{Q}}_{0}=\overline{\mathbf{F}}
$$

where $\overline{\mathbf{M}}, \overline{\mathbf{C}}$, and $\overline{\mathbf{K}}$ are obtained from $\widetilde{\mathbf{M}}, \widetilde{\mathbf{C}}$, and $\widetilde{\mathbf{K}}$ by deleting the 1 to $2 k$ rows and the 1 to $2 k$ columns and $\overline{\mathbf{Q}}_{0}$ is obtained from $\mathbf{Q}_{0}$ by deleting the 1 to $2 k$ row. And $\overline{\mathbf{F}}$ is obtained form $\widetilde{\mathbf{F}}$ by the operation that

$$
\begin{aligned}
\overline{\mathbf{F}}_{a}= & \widetilde{\mathbf{F}}_{a+2 k}-\sum_{p=1}^{k} \widetilde{\mathbf{M}}_{a+2 k, 2 p-1} \ddot{e}_{p}(t)-\sum_{p=1}^{k} \widetilde{\mathbf{C}}_{a+2 k, 2 p-1} \dot{e}_{p}(t) \\
& -\sum_{p=1}^{k} \widetilde{\mathbf{K}}_{a+2 k, 2 p-1} e_{p}(t),
\end{aligned}
$$

where $a=1,2, \ldots, 4 n k+3-2 k$.

2.3. Rope Slack Condition. Now consider a conveyance hoisted by two ropes, the dynamics of the system will be more complex if one of ropes is slack due to the deviation of drums. One rope is completely slack or tight which is judged by the tension at the connection point of the conveyance. Before the rope is completely slack, the dynamic response can be obtained by the equations derived before. But, if the slack rope is completely slack, the constraint condition between ropes and conveyance will change.

When one rope is slack, it will not bear loads from the conveyance while the torsion still exists. Then, the dynamic torsional displacements of lower ends of ropes can be expressed by the rotation angle of conveyance as

$$
g_{i}=\theta_{i, 2 n+1}-\theta_{c}=0, \quad i=1,2 .
$$

If rope 1 was slack, the relationship between dynamic longitudinal displacement of conveyance and ropes at lower ends can be given as

$$
g_{3}=\varphi_{c}+\frac{2}{d}\left(u_{2,2 n+1}-u_{c}\right)=0 .
$$




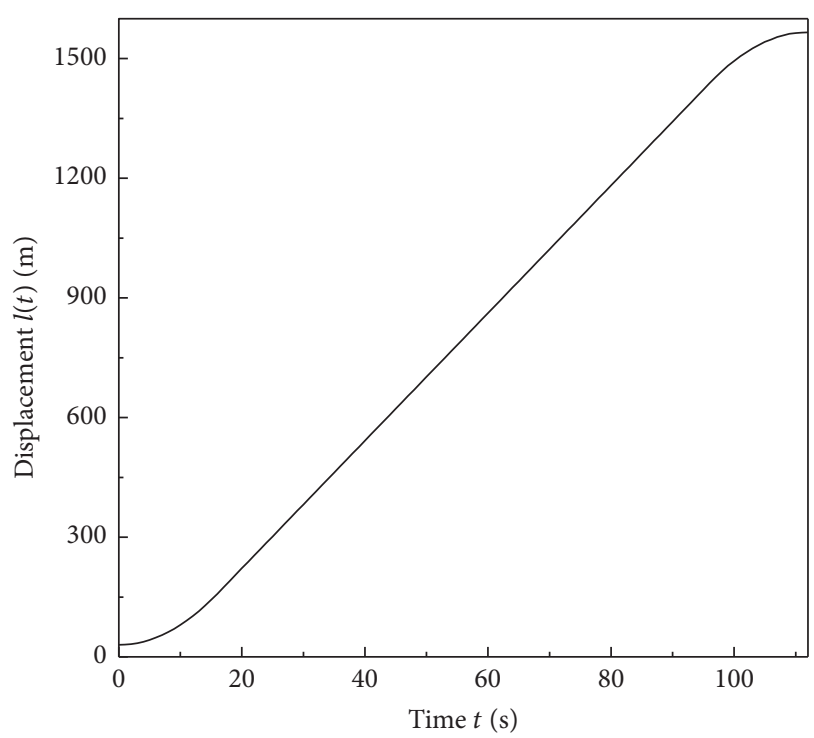

(a)

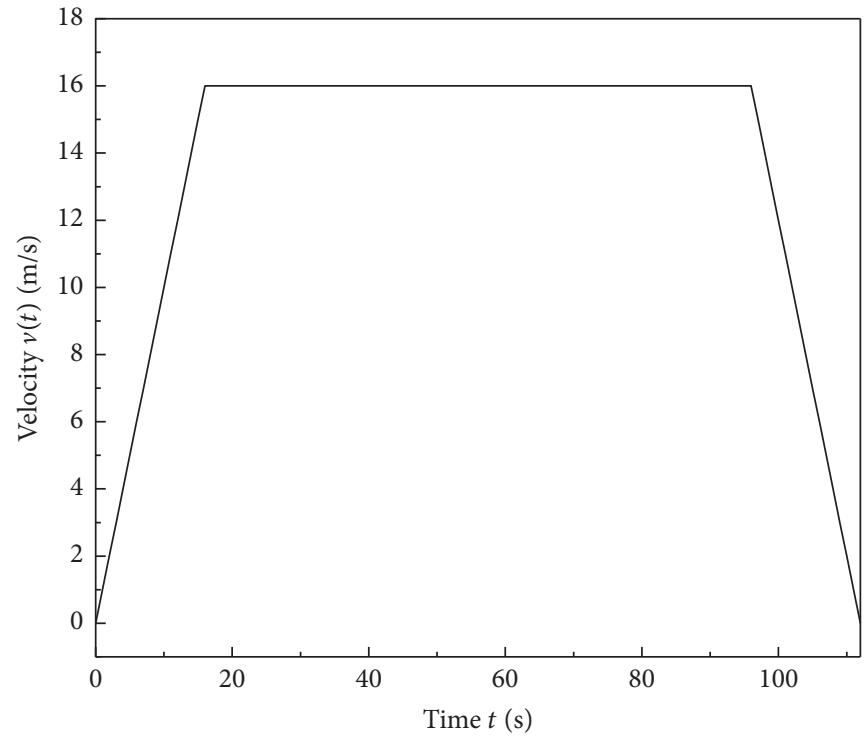

(b)

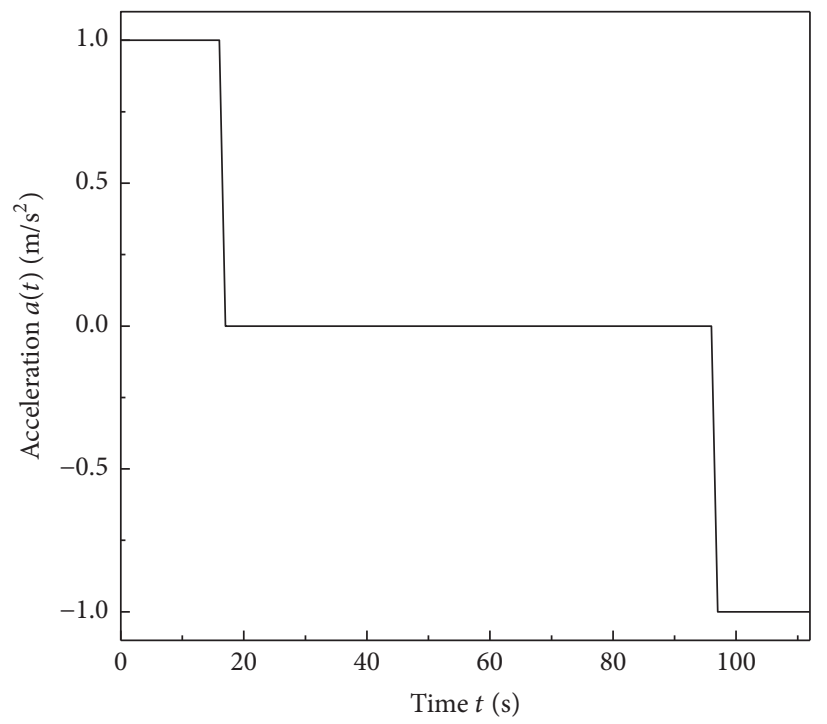

(c)

FIGURE 4: Prescribed movement profiles.

For the convenient of dealing with the constraint condition, the sequence of elements in $\mathbf{Q}$ is transformed, and the expression of $\mathbf{G}_{0}$ can be expressed as

$$
\begin{aligned}
& \mathbf{G}_{01,4(2 n)+2}=\mathbf{G}_{02,4(2 n)+2}=-1, \\
& \mathbf{G}_{03,4(2 n)+3}=-\mathbf{G}_{03,4(2 n)+4}=\frac{2}{d} .
\end{aligned}
$$

The process of transforming DAEs to ODEs and obtaining the solution is the same as above, and it could be solved by Newmark- $\beta$ method.

\section{Results and Analysis}

3.1. Parameters. In mine hoisting system, travelling distance ranges is large and continuously being increased. For example, in the South African gold mining industry, the depth of Kloof Gold Mine shaft is $2085 \mathrm{~m}$, and the hoisting distance of South Deep Gold Mine has reached $3000 \mathrm{~m}$. Now in China, new mines with shaft depths in the region of 1000 $2000 \mathrm{~m}$, based on parallel hoisting system, have recently been considered, for example, mine shafts in Chihong and Linyi. In this section, two-rope parallel hoisting system is discussed. The initial and maximum lengths of ropes are reset as $30 \mathrm{~m}$ and $1566 \mathrm{~m}$, respectively. The downward movement profiles are shown in Figure 4. 
TABLE 1: System parameters used in calculation.

\begin{tabular}{lc}
\hline Parameter & Value \\
\hline$\rho$ & $11.5 \mathrm{~kg} / \mathrm{m}$ \\
$J$ & $4.518 \times 10^{-3} \mathrm{~kg} \cdot \mathrm{m}^{2}$ \\
$Q_{1}^{a}, Q_{2}^{a}$ & $1.746 \times 10^{8} \mathrm{~N}$ \\
$Q_{2}^{d}, Q_{2}^{d}$ & $1.227 \times 10^{4} \mathrm{~N} \cdot \mathrm{m}^{2}$ \\
$-Q_{1}^{b}, Q_{2}^{b}$ & $8.539 \times 10^{5} \mathrm{~N} \cdot \mathrm{m}$ \\
$-Q_{1}^{c}, Q_{2}^{c}$ & $7.969 \times 10^{5} \mathrm{~N} \cdot \mathrm{m}$ \\
$\zeta_{u}, \zeta_{\theta}$ & $1.3 \times 10^{-4}$ \\
$d$ & $0.35 \mathrm{~m}$ \\
$m_{c}$ & $20 \mathrm{t}$ \\
$J_{c x}$ & $8.313 \times 10^{3} \mathrm{~kg} \cdot \mathrm{m}^{2}$ \\
$J_{c z}$ & $1.155 \times 10^{4} \mathrm{~kg} \cdot \mathrm{m}^{2}$ \\
$k_{c}$ & $8 \times 10^{5} \mathrm{~N} / \mathrm{m}$ \\
$c_{c}$ & $6.7 \times 10^{4} \mathrm{Ns} / \mathrm{m}$ \\
$r_{1}$ & $1.1 \mathrm{~m}$ \\
$r_{2}$ & $0.8 \mathrm{~m}$ \\
$R$ & $2.5 \mathrm{~m}$ \\
\hline
\end{tabular}

Following the regulations that are mentioned above for parameter symbols, system parameters used in calculation are listed in Table 1, in which $R$ is the radius of drum.

The longitudinal drive deviation $e_{i}(t)$ on $i$ th rope could be denoted as

$$
e_{i}(t)=e s_{i}(t)+e r_{i}(t), \quad i=1,2,
$$

where $e s_{1}(t)=e_{S} \sin \left(n_{1} \cdot\left(l(t)-l_{0}\right) / R\right)$ and $e s_{2}(t)=e_{S} \sin \left(n_{2}\right.$. $\left.\left(l(t)-l_{0}\right) / R+\alpha\right)$ denote swing amplitude of noncircular drum, respectively. The $e_{S}$ and $\alpha$ are expressed as $e_{S}=\kappa \cdot R$ and $\alpha=\varsigma \cdot \pi$, in which $\kappa$ and $\varsigma$ are scale coefficients; in this paper, $\kappa=0.001$ and $\varsigma=0$. The coefficients $n_{1}$ and $n_{2}$ denote degree of defect distribution, respectively; in this paper, $n_{1}=1$ and $n_{2}=2 . e r_{1}(t)=0$ and $e r_{2}(t)=\left(e_{r} / R\right)\left(l(t)-l_{0}\right)$ denote relative deviation induced by radius' error of drum, respectively. The $e_{r}$ is expressed as $e_{r}=\xi \cdot R$, in which $\xi$ is a scale coefficient.

3.2. Dynamic Response under Drive Deviation. The dynamic responses of the hoisting system are affected by many factors, but the influence of the radius' error of the hoisting rope $e_{r}$ is more important because it brings large tension difference. In this paper, the following parametric study will concentrate on the influence of the radius' error of the hoisting rope $e_{r}$ and the dynamic responses are investigated. In addition, the variable-domain finite element number $n=5$ is used for the convergence of the solution, and the results are shown in Figures 5 8.

As shown in Figures 5 7, the tension and torsion of both ropes fluctuate greatly at the beginning because of hoisting acceleration, and the fluctuation decreases with the hoisting velocity at constant speed, but resonances occur while the natural frequency is close to the excitation frequency, which is shown in Figure 8. It is obvious that the longitudinal and torsional frequencies about hoisting ropes system decrease as the length increases, but the rotational frequencies about conveyance keep almost unchanged. Intersection points between

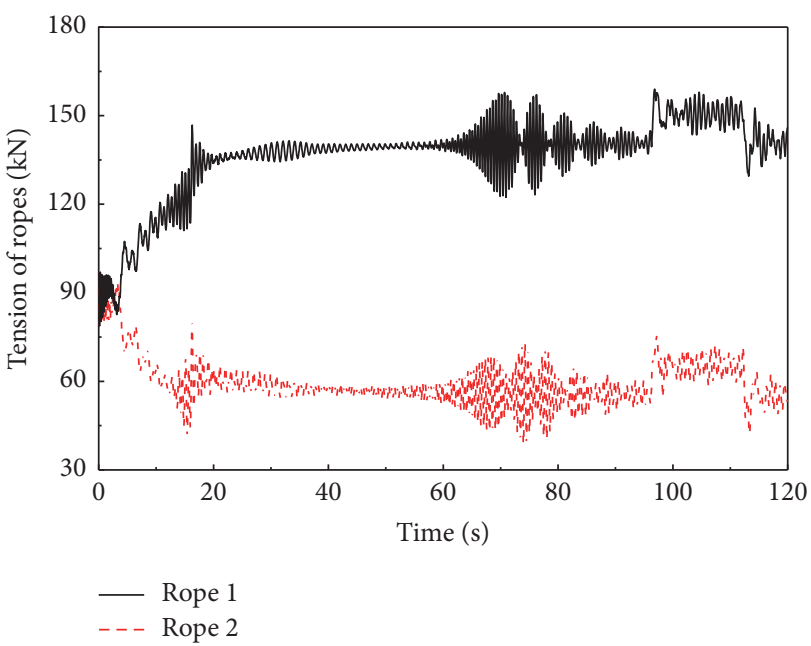

(a)

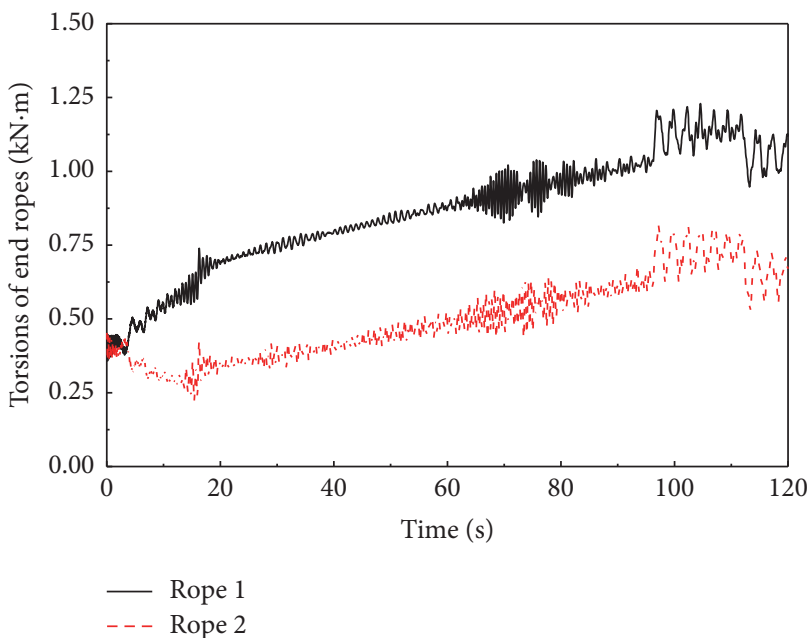

(b)

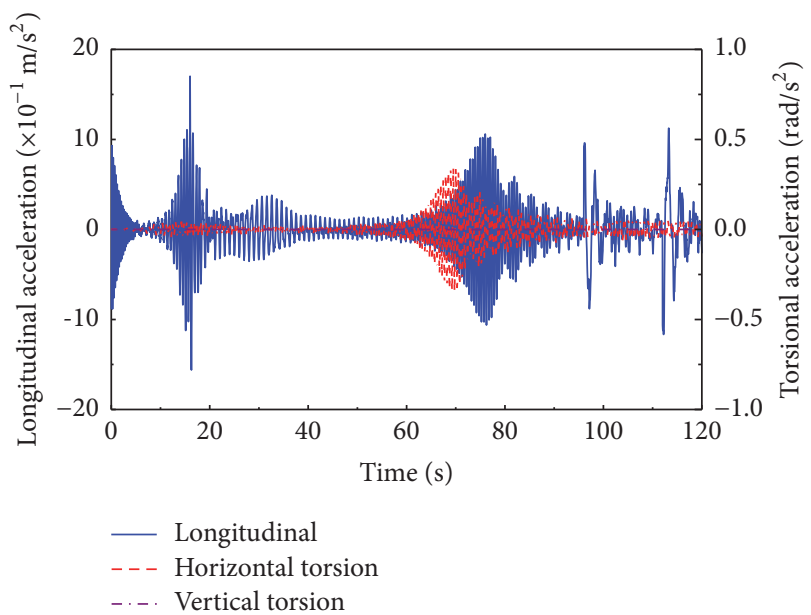

(c)

FIgURE 5: (a) Tensions, (b) torsions of the end ropes, and (c) accelerations of conveyance, calculated using $\xi=0.0005$.

the natural frequencies and the excitation frequencies denote the resonance locations. It should be noted that there are 

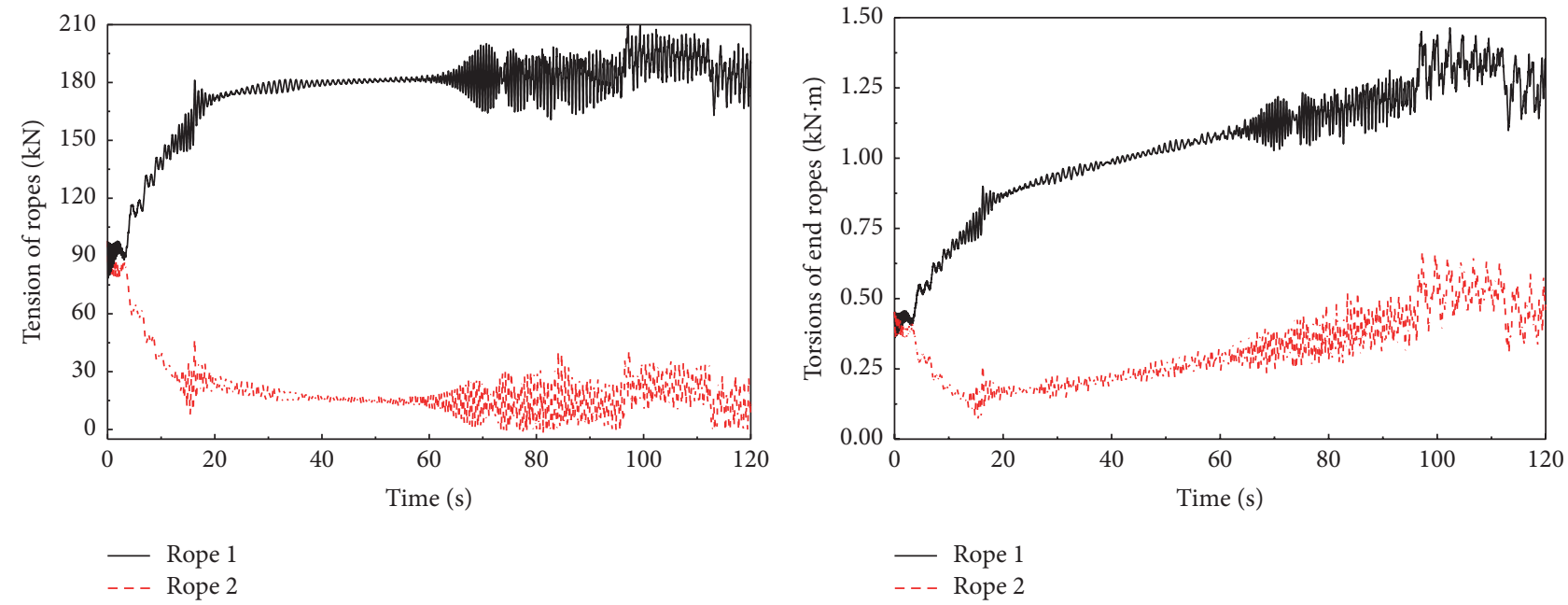

(a)

(b)

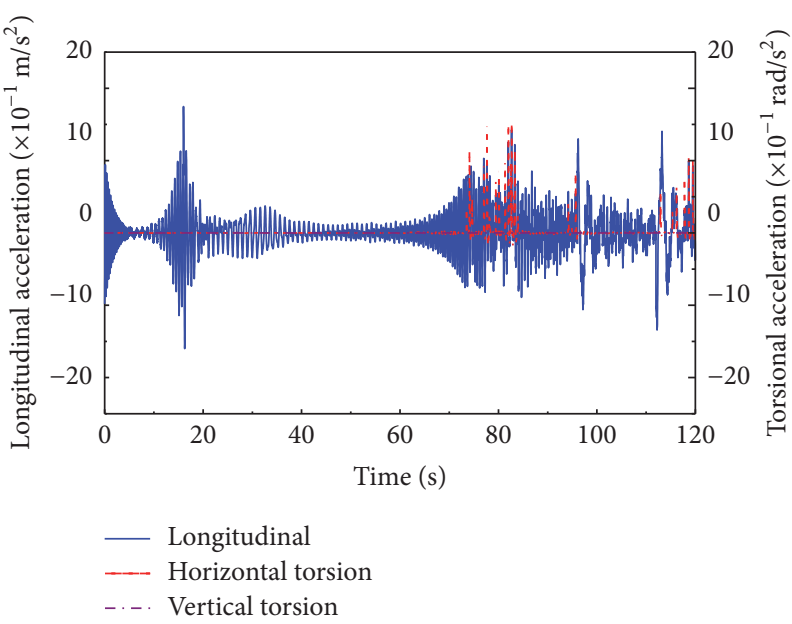

(c)

FIgURE 6: (a) Tensions, (b) torsions of the end ropes, and (c) accelerations of conveyance, calculated using $\xi=0.001$.

multiple resonance locations during time interval $60 \sim 90 \mathrm{~s}$, which validate the results of Figures 5 7. Although the torsion of ropes has approximately the same trend of vibration with tension in the beginning, there is some difference that the torsions of two ropes increase even if the tension of one rope drops gradually or the rope is slack. The reason for this phenomenon is that the weight of rope along varying length goes up and the tension of upper ends of rope rises gradually; therefore the torsions of the two ropes move up which also can be explained by (5) and (7). The accelerations of conveyance change violently in the horizontal direction during one rope alternating between the slack and tight states.

The dynamic tension difference between the ropes with the coefficient $\xi=0.0015$ (Figure 7) is changed more than that with the coefficient $\xi=0.001$ (Figure 6) and $\xi=0.0005$ (Figure 5), which indicates that the deviation between ropes caused by the winding radius' error of drum can bring large influence to the tension difference. It is obvious that even if the deviation was only $1 / 1000$ of the radius, the tension of one rope would dramatically drop, even close to zero, which means that there is an obvious tension imbalance between two ropes. It is worth noting that intermittent tension of one rope would happen and last for a long time, which is shown in Figure 6(a), if the deviation (for example, 1/1000 of the radius) was inappropriate. It should be emphasized that unreasonable discordance between ropes would bring tension imbalance and should be controlled during the design and manufacture of drum, especially in super deep parallel hoisting system.

\section{Conclusions}

This paper addresses the modeling of parallel hoisting system considering the drive deviation, and the Vd-FEM and Lagrange's equations of the first kind are used to derive the equations of motion of the system. The dynamic model using Vd-FEM proposed for parallel hoisting system can be readily utilized to solve several challenging problems, including the slack or tight condition of ropes, the tensions and torsions between conveyance and ropes, and the rotation of the conveyance. 

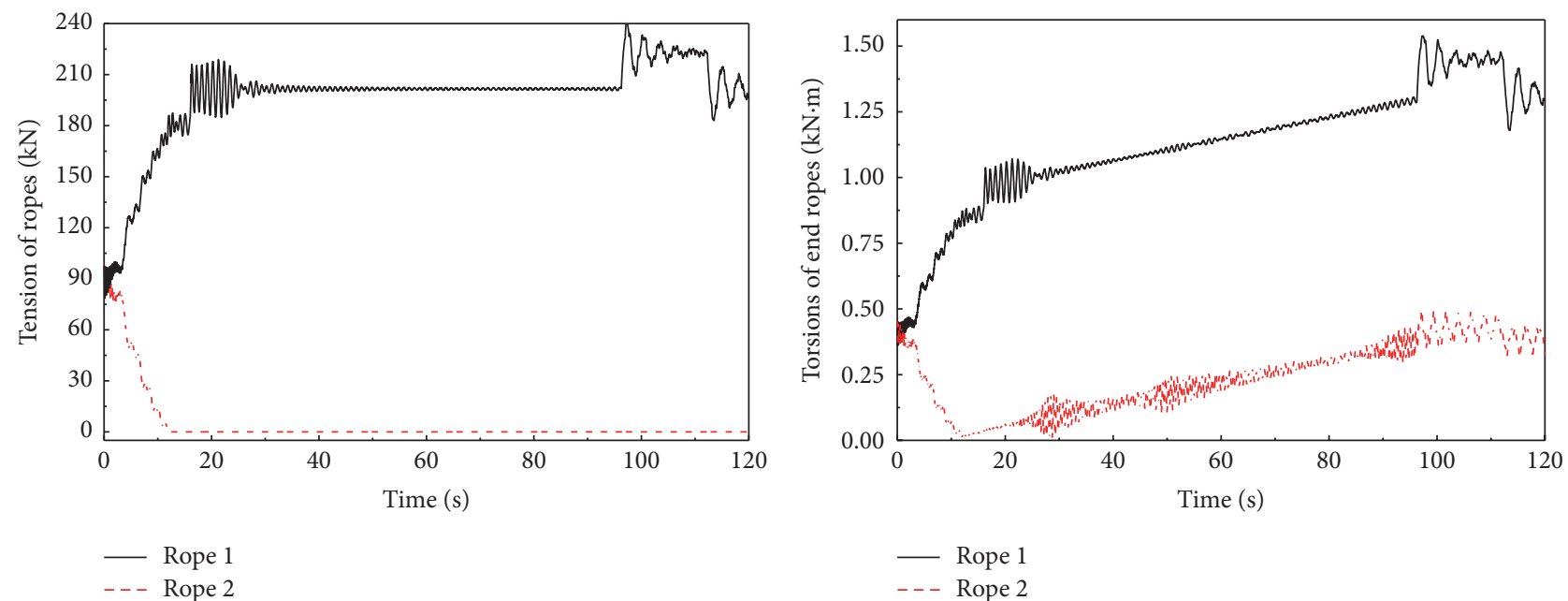

(a)

(b)

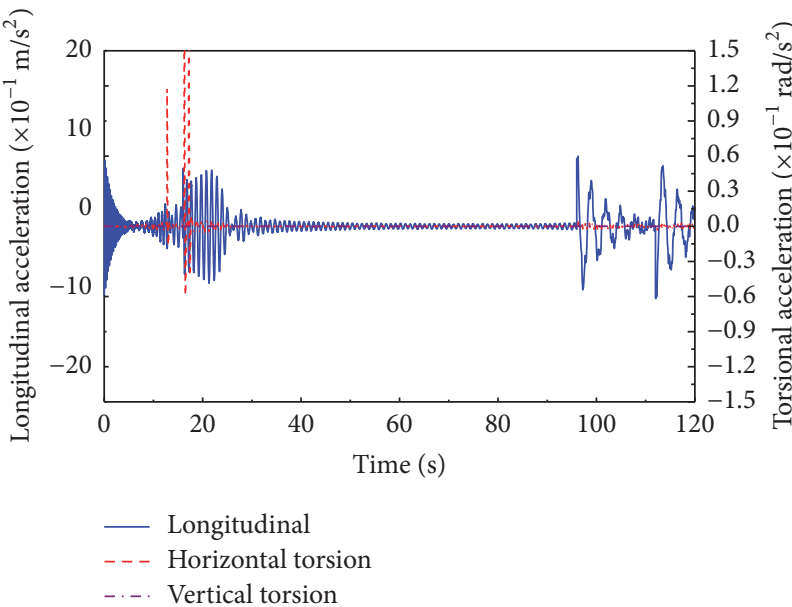

(c)

Figure 7: (a) Tensions, (b) torsions of the end ropes, and (c) accelerations of conveyance, calculated using $\xi=0.0015$.

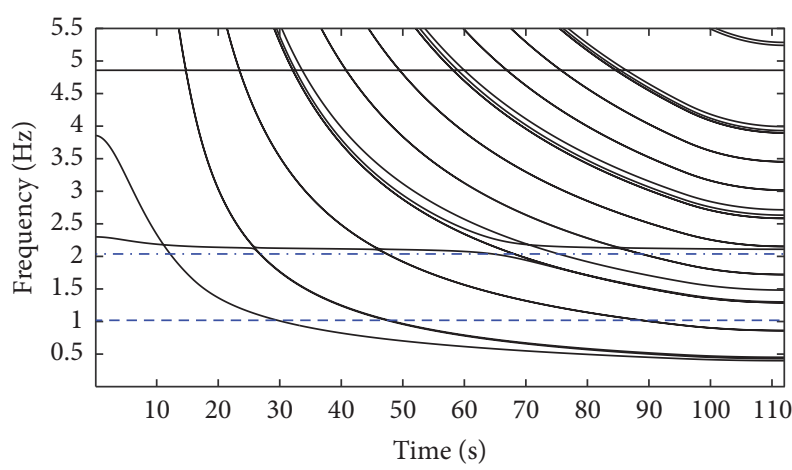

- - Excitation frequency 1

-... Excitation frequency 2

Figure 8: Longitudinal, torsional, and excitation frequencies.

It should be noted that the torsions of two ropes increase even if the tension of one rope drops gradually or the rope is slack. The drive deviation (only 1/1000 of the radius) between ropes caused by the winding radius' error of drum will bring large influence to the tension difference and even lead to slack rope. And unreasonable discordance between ropes should be controlled for the design and manufacturing of drum on super deep parallel hoisting system.

\section{Appendix}

The matrix of element mass, damping, stiffness, and force is listed as follows:

$$
\begin{aligned}
\mathbf{m}_{i, j}= & \rho \mathbf{A}_{1}, \\
\mathbf{c}_{i, j}= & \rho\left(2 \mathbf{A}_{3}+2 v \mathbf{A}_{2}^{T}-v \mathbf{A}_{2}\right)+\zeta_{u} Q_{i}^{a} \mathbf{A}_{2}^{T}+\kappa \rho v \mathbf{A}_{11}, \\
\mathbf{k}_{u i, j} & \\
= & \rho\left(\mathbf{A}_{6}+2 v \mathbf{A}_{7}+a \mathbf{A}_{2}^{T}+v^{2} \mathbf{A}_{14}-v^{2} \mathbf{A}_{4}-v \mathbf{A}_{5}\right) \\
& +Q_{i}^{a} \mathbf{A}_{4}+\zeta_{u} Q_{i}^{a} \mathbf{A}_{7}+\kappa\left(\rho v \mathbf{A}_{12}+\rho v^{2} \mathbf{A}_{13}\right),
\end{aligned}
$$




$$
\begin{aligned}
\mathbf{c}_{u i \theta i, j}= & \zeta_{u} Q_{i}^{b} \mathbf{A}_{2}^{T}, \\
\mathbf{k}_{u i \theta i, j}= & 0.5\left(Q_{i}^{b}+Q_{i}^{c}\right) \mathbf{A}_{4}+\zeta_{u} Q_{i}^{b} \mathbf{A}_{7}, \\
\mathbf{f}_{u i, j} & \\
= & \rho v^{2} \mathbf{A}_{9}+\rho(g-a) \mathbf{A}_{8}-\left(\rho g l+\frac{m_{c} g}{k}\right) \mathbf{A}_{9} \\
& +\rho g \mathbf{A}_{15}-\kappa \rho v^{2} \mathbf{A}_{10}, \\
\mathbf{m}_{\theta i, j}= & J \mathbf{A}_{1}, \\
\mathbf{c}_{\theta i, j}= & J\left(2 \mathbf{A}_{3}+2 v \mathbf{A}_{2}^{T}-v \mathbf{A}_{2}\right)+\zeta_{\theta} Q_{i}^{d} \mathbf{A}_{2}^{T}+\kappa J v \mathbf{A}_{11}, \\
\mathbf{k}_{\theta i, j} & \\
= & J\left(\mathbf{A}_{6}+2 v \mathbf{A}_{7}+a \mathbf{A}_{2}^{T}+v^{2} \mathbf{A}_{20}-v^{2} \mathbf{A}_{4}-v \mathbf{A}_{5}\right) \\
& +Q_{i}^{d} \mathbf{A}_{4}+\zeta_{\theta} Q_{i}^{d} \mathbf{A}_{7}+\kappa\left(J v \mathbf{A}_{12}+J v^{2} \mathbf{A}_{13}\right), \\
\mathbf{c}_{\theta i u i, j}= & \zeta_{\theta} Q_{i}^{c} \mathbf{A}_{2}^{T}, \\
\mathbf{k}_{\theta i u i, j}= & 0.5\left(Q_{i}^{b}+Q_{i}^{c}\right) \mathbf{A}_{4}+\zeta_{\theta} Q_{i}^{c} \mathbf{A}_{7}, \\
\mathbf{f}_{\theta i, j}= & \frac{\left[-\left(m_{c} / k+\rho l\right) g \mathbf{A}_{9}+\rho g \mathbf{A}_{15}\right] Q_{i}^{c}}{Q_{i}^{a}},
\end{aligned}
$$

where

$$
\kappa= \begin{cases}1, & j=n \\ 0, & \text { others. }\end{cases}
$$

The matrixes of coefficients are as follows:

$$
\begin{aligned}
& \mathbf{A}_{1}=\int_{(j-1) l(t) / n}^{j l(t) / n} \mathbf{N}_{j}^{T} \mathbf{N}_{j} \mathrm{~d} x, \\
& \mathbf{A}_{2}=\int_{(j-1) l(t) / n}^{j l(t) / n} \mathbf{N}_{j, x}^{T} \mathbf{N}_{j} \mathrm{~d} x,
\end{aligned}
$$

$$
\begin{aligned}
\mathbf{A}_{3} & =\int_{(j-1) l(t) / n}^{j l(t) / n} \mathbf{N}_{j}^{T} \mathbf{N}_{j, t} \mathrm{~d} x, \\
\mathbf{A}_{4} & =\int_{(j-1) l(t) / n}^{j l(t) / n} \mathbf{N}_{j, x}^{T} \mathbf{N}_{j, x} \mathrm{~d} x, \\
\mathbf{A}_{5} & =\int_{(j-1) l(t) / n}^{j l(t) / n} \mathbf{N}_{j, x}^{T} \mathbf{N}_{j, t} \mathrm{~d} x, \\
\mathbf{A}_{6} & =\int_{(j-1) l(t) / n}^{j l(t) / n} \mathbf{N}_{j}^{T} \mathbf{N}_{j, t t} \mathrm{~d} x, \\
\mathbf{A}_{7} & =\int_{(j-1) l(t) / n}^{j l(t) / n} \mathbf{N}_{j}^{T} \mathbf{N}_{j, t x} \mathrm{~d} x, \\
\mathbf{A}_{8} & =\int_{(j-1) l(t) / n}^{j l(t) / n} \mathbf{N}_{j}^{T} \mathrm{~d} x, \\
\mathbf{A}_{9} & =\int_{(j-1) l(t) / n}^{j l(t) / n} \mathbf{N}_{j, x}^{T} \mathrm{~d} x, \\
\mathbf{A}_{10} & =\left.\mathbf{N}_{n}^{T}\right|_{x=l(t)}, \\
\mathbf{A}_{11} & =\left.\mathbf{N}_{n}^{T} \mathbf{N}_{n}\right|_{x=l(t)}, \\
\mathbf{A}_{12} & =\left.\mathbf{N}_{n}^{T} \mathbf{N}_{n, t}\right|_{x=l(t)}, \\
\mathbf{A}_{13} & =\left.\mathbf{N}_{n}^{T} \mathbf{N}_{n, x}\right|_{x=l(t)},
\end{aligned}
$$

$$
\begin{aligned}
\mathbf{A}_{14} & =\int_{(j-1) l(t) / n}^{j l(t) / n} \mathbf{N}_{j}^{T} \mathbf{N}_{j, x x} \mathrm{~d} x, \\
\mathbf{A}_{15} & =\int_{(j-1) l(t) / n}^{j l(t) / n} x \mathbf{N}_{j, x}^{T} \mathrm{~d} x,
\end{aligned}
$$

where the derivatives of shape function about $t$ and $x$ are given as

$$
\begin{aligned}
\mathbf{N}_{j, x} & =\left[\begin{array}{lll}
\frac{4 n^{2} x}{l^{2}}-\frac{n(4 j-1)}{l} & \left.\frac{4 n(2 j-1)}{l}-\frac{8 n^{2} x}{l^{2}} \frac{4 n^{2} x}{l^{2}}-\frac{n(4 j-3)}{l}\right]
\end{array}\right] \\
\mathbf{N}_{j, x x} & =\frac{4 n^{2}}{l^{2}}\left[\begin{array}{lll}
1 & -2 & 1
\end{array}\right], \\
\mathbf{N}_{j, t} & =\frac{v n x}{l^{3}}\left[\begin{array}{ll}
(4 j-1) l-4 n x & 8 n x-4(2 j-1) l(4 j-3) l-4 n x
\end{array}\right], \\
\mathbf{N}_{j, x t} & =\frac{n v}{l^{3}}\left[\left(\begin{array}{ll}
(4-1) l-8 n x & 16 n x-4(2 j-1) l(4 j-3) l-8 n x
\end{array}\right],\right. \\
\mathbf{N}_{j, t t} & =\frac{n x}{l^{4}}\left[\begin{array}{c}
-l^{2} a+2 l v^{2}+4 l^{2} a j-8 l j v^{2}+12 n v^{2} x-4 \operatorname{lan} x \\
4\left(l^{2} a-2 l v^{2}-2 l^{2} a j+4 l j v^{2}-6 n v^{2} x+2 \operatorname{lan} x\right) \\
\left(-3 l^{2} a+6 l v^{2}+4 l^{2} a j-8 l j v^{2}+12 n v^{2} x-4 \operatorname{lan} x\right)
\end{array}\right]^{\mathrm{T}}
\end{aligned}
$$




\section{Competing Interests}

The authors declare that they have no competing interests.

\section{Acknowledgments}

This work is supported by the National Key Basic Research Program of China (2014CB049401), the National Natural Science Foundation of China (51475456), the Fundamental Research Funds for the Central Universities (2014YC06), and the Priority Academic Program Development of Jiangsu Higher Education Institutions (PAPD).

\section{References}

[1] J. Du, H. Bao, C. Cui, and D. Yang, "Dynamic analysis of cabledriven parallel manipulators with time-varying cable lengths," Finite Elements in Analysis and Design, vol. 48, no. 1, pp. 13921399, 2012.

[2] W. D. Zhu and J. Ni, "Energetics and stability of translating media with an arbitrarily varying length," Journal of Vibration and Acoustics, Transactions of the ASME, vol. 122, no. 3, pp. 295304, 2000.

[3] S. Kaczmarczyk and W. Ostachowicz, "Transient vibration phenomena in deep mine hoisting cables. Part 1: mathematical model," Journal of Sound and Vibration, vol. 262, no. 2, pp. 219244, 2003.

[4] J. Wang, G. Cao, Z. Zhu, Y. Wang, and W. Peng, "Lateral response of cable-guided hoisting system with time-varying length: theoretical model and dynamics simulation verification," Proceedings of the Institution of Mechanical Engineers Part C: Journal of Mechanical Engineering Science, vol. 229, no. 16, pp. 2908-2920, 2015.

[5] W. D. Zhu and H. Ren, "An accurate spatial discretization and substructure method with application to moving elevator cablecar systems-part I: methodology," Journal of Vibration and Acoustics, Transactions of the ASME, vol. 135, no. 5, 10 pages, 2013.

[6] S. Kawamura, H. Kino, and C. Won, "High-speed manipulation by using parallel wire-driven robots," Robotica, vol. 18, no. 1, pp. 13-21, 2000.

[7] E. Ottaviano, "Analysis and design of a four-cable-driven parallel manipulator for planar and spatial tasks," Proceedings of the Institution of Mechanical Engineers, Part C: Journal of Mechanical Engineering Science, vol. 222, no. 8, pp. 1583-1592, 2008.

[8] B. Y. Duan, Y. Y. Qiu, F. S. Zhang, and B. Zi, "On design and experiment of the feed cable-suspended structure for super antenna," Mechatronics, vol. 19, no. 4, pp. 503-509, 2009.

[9] M. H. Korayem and M. Bamdad, "Dynamic load-carrying capacity of cable-suspended parallel manipulators," The International Journal of Advanced Manufacturing Technology, vol. 44, no. 7-8, pp. 829-840, 2009.

[10] X.-G. Shao, Z.-C. Zhu, Q.-G. Wang, P. C. Chen, B. Zi, and G.-H. Cao, "Non-smooth dynamical analysis and experimental validation of the cable-suspended parallel manipulator," Proceedings of the Institution of Mechanical Engineers, Part C: Journal of Mechanical Engineering Science, vol. 226, no. 10, pp. 2456-2466, 2012.
[11] Y. Wang, G. Cao, Z. Zhu, J. Wang, and N. Wang, "1483. Longitudinal response of parallel hoisting system with timevarying rope length," Journal of Vibroengineering, vol. 16, no. 8, pp. 4088-4101, 2014.

[12] O. C. Zienkiewicz and R. L. Taylor, The Finite Element Method for Solid and Structural Mechanics, Butterworth-Heinemann, 2005.

[13] L. Meirovitch, Principles and Techniques of Vibrations, Prentice Hall, Upper Saddle River, NJ, USA, 1997.

[14] M. Stylianou and B. Tabarrok, "Finite element analysis of an axially moving beam, part I: time integration," Journal of Sound and Vibration, vol. 178, no. 4, pp. 433-453, 1994.

[15] M. Stylianou and B. Tabarrok, "Finite element analysis of an axially moving beam, part II: stability analysis," Journal of Sound and Vibration, vol. 178, no. 4, pp. 455-481, 1994.

[16] P.-H. Wang, R.-F. Fung, and M.-J. Lee, "Finite element analysis of a three-dimensional underwater cable with time-dependent length," Journal of Sound and Vibration, vol. 209, no. 2, pp. 223249, 1998.

[17] K. A. F. Moustafa, E. H. Gad, A. M. A. El-Moneer, and M. I. S. Ismail, "Modelling and control of overhead cranes with flexible variable-length cable by finite element method," Transactions of the Institute of Measurement and Control, vol. 27, no. 1, pp. 1-20, 2005.

[18] G. A. Costello, Theory of Wire Rope, Springer Science \& Business Media, Berlin, Germany, 1997.

[19] J. M. Hamilton, "Vibration-based techniques for measuring the elastic properties of ropes and the added mass of submerged objects," Journal of Atmospheric and Oceanic Technology, vol. 17, no. 5, pp. 688-697, 2000.

[20] S. K. Soltakhanov, M. P. Yushkov, and S. A. Zegzhda, Mechanics of Non-Holonomic Systems: A New Class of Control Systems, Foundations of Engineering Mechanics, Springer Science \& Business Media, Berlin, Germany, 2009. 


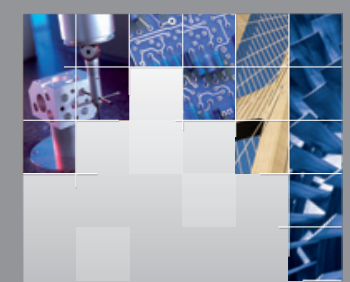

\section{Enfincering}
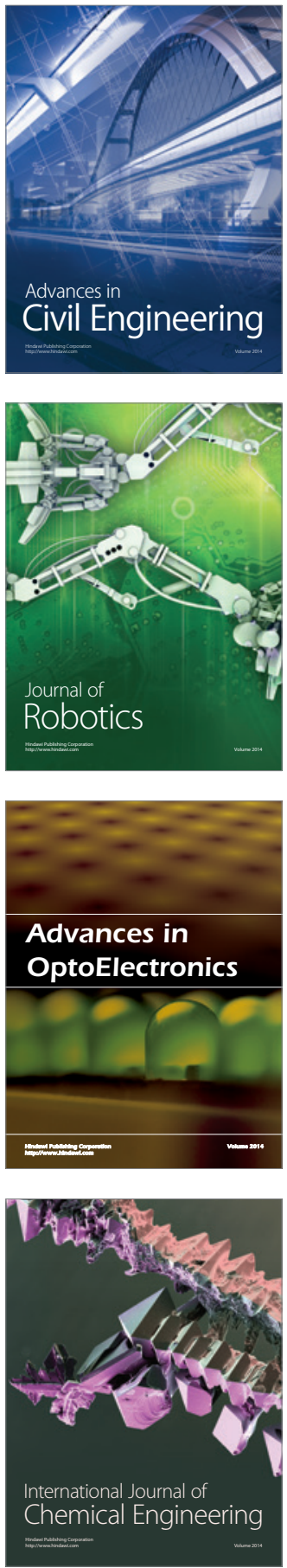

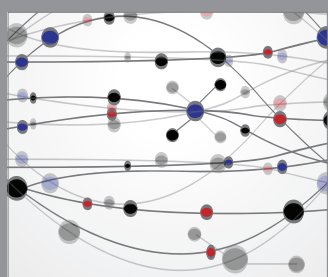

The Scientific World Journal

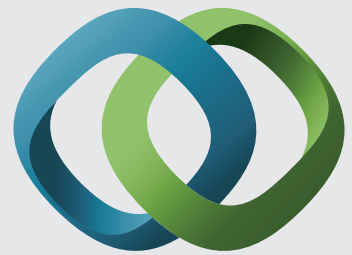

\section{Hindawi}

Submit your manuscripts at

https://www.hindawi.com
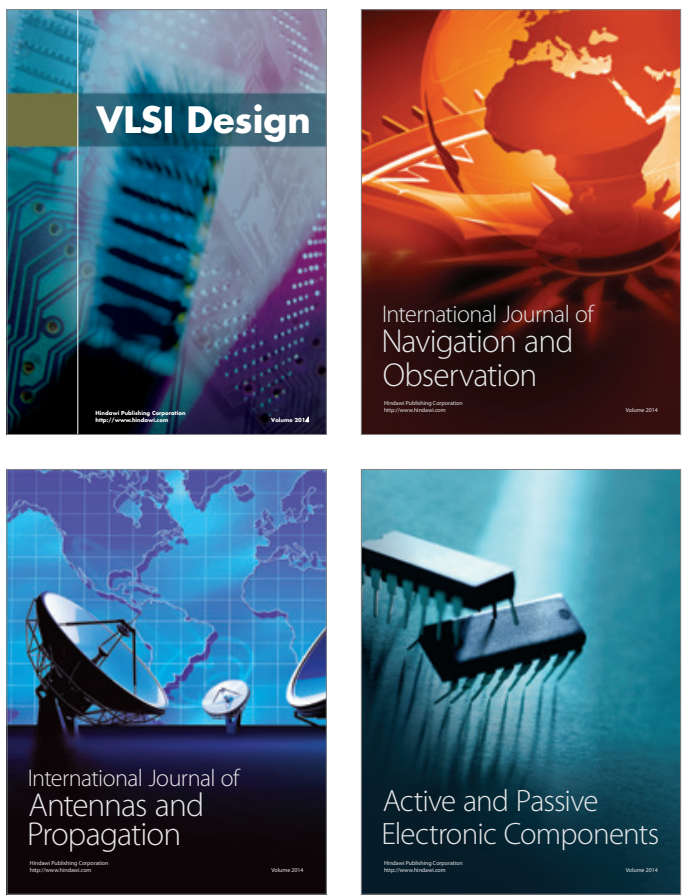
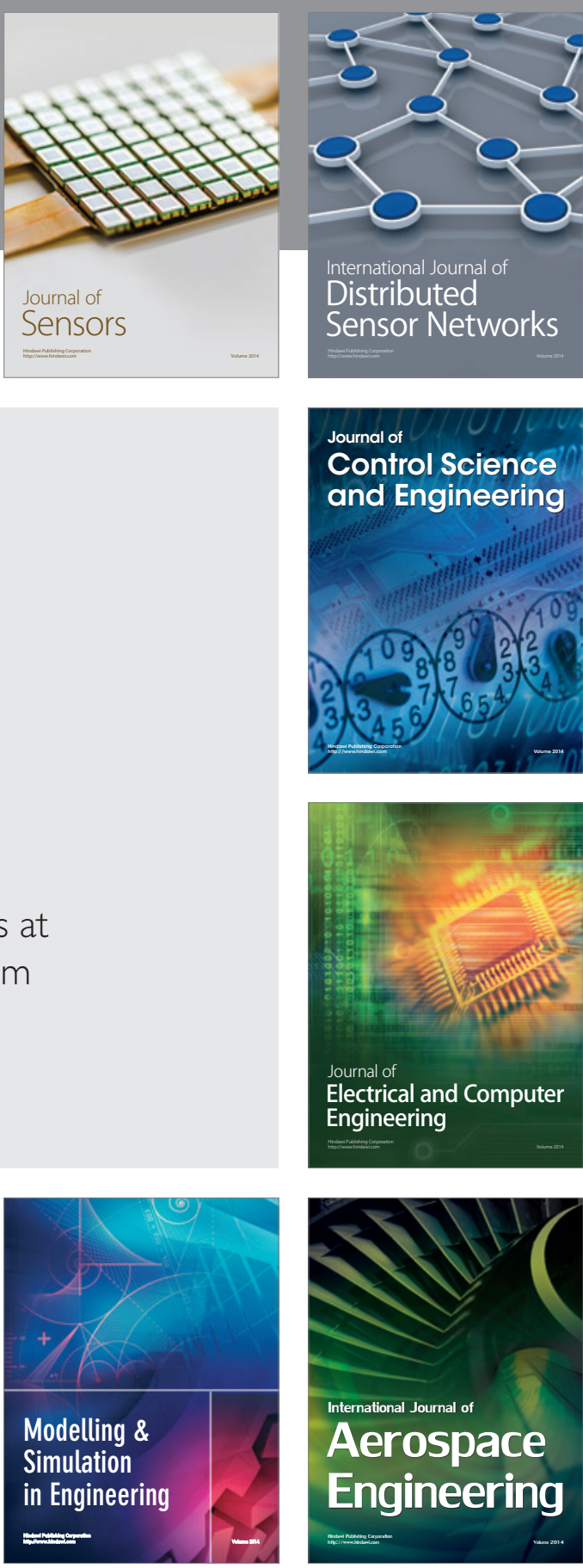

International Journal of

Distributed

Sensor Networks

$-$

Joumal of

Control Science

and Engineering
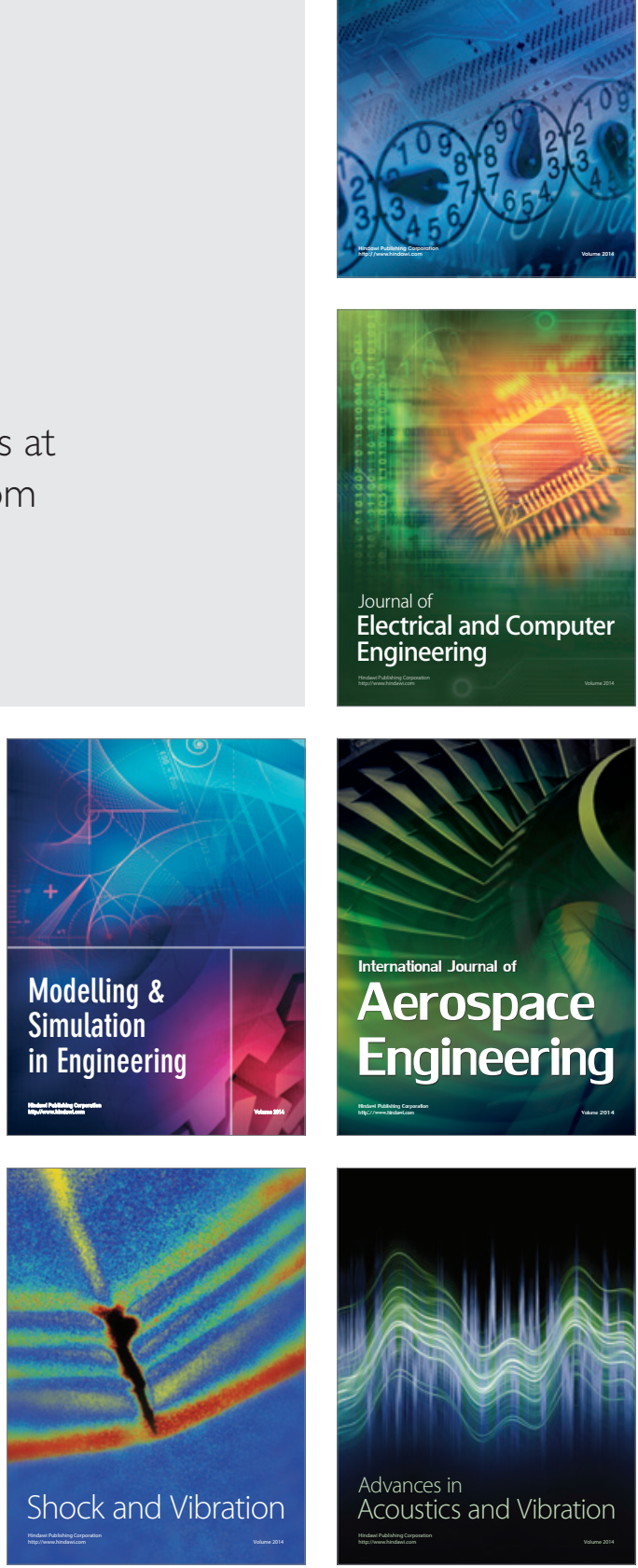